\title{
Research of gestational diabetes mellitus risk evaluation method
}

\author{
Zhenyu Chi ${ }^{\mathrm{a}}$, Song Zhang ${ }^{\mathrm{a}}$, Yang Wang ${ }^{\mathrm{b}}$, Lin Yang ${ }^{\mathrm{a}, *}$, Yimin Yang ${ }^{\mathrm{a}}$ and Xuwen $\mathrm{Li}^{\mathrm{a}}$ \\ ${ }^{a}$ College of Life Science and Bio-engineering, Beijing University of Technology, Beijing, China \\ ${ }^{\mathrm{b}}$ Shenzhen Huada Gene Research Institute, Shenzhen, China
}

\begin{abstract}
.
BACKGROUND: Gestational diabetes mellitus (GDM) is not easily detected. The difficulty in detecting GDM is largely due to the late onset of clinical symptoms as well as the various complications that result from GDM [1].

OBJECTIVE: GDM greatly influences both mother and child. Therefore, the purpose of this study was to reduce the morbidity of GDM.

METHODS: In this study, risk factors that influence GDM were selected through statistical analysis. Multivariable logistic regression analysis was used to obtain the regression equation and Odds Ratio (OR) value. The risk score of each factor was obtained according to the OR value.

RESULTS: The score of every pregnant woman could be very intuitively used to show the risk of getting GDM.

CONCLUSION: Through the above methods, a comprehensive risk evaluation method of detecting GDM was developed.
\end{abstract}

Keywords: Gestational diabetes mellitus, logistic regression, risk factors

\section{Introduction}

Gestational diabetes mellitus (GDM) is defined as when a gravida's glucose metabolism is discovered to be at an abnormally high level during pregnancy despite not having been previously diagnosed with diabetes [2,3]. While pregnant, if the blood glucose cannot be effectively controlled, the incidence rate of gravidas' complications will increase greatly. In addition, gravidas' high blood glucose is capable of directly influencing perinatal health [4-7]. While there are many domestic and foreign studies about the risk factors of GDM, these studies are limited because they only analyze a single factor or just a few factors [8-11]. This study aimed to develop an comprehensive method to evaluate GDM. The method can help doctors determine the presence of GDM earlier.

\section{Methods and results}

In this research, a retrospective case control study was used to locate the data of 836 pregnant women who had complete prenatal examination data and gave birth in a Beijing hospital between 2007 and 2009.

\footnotetext{
${ }^{*}$ Corresponding author: Lin Yang, No.100 Pingleyuan, Chaoyang District, Beijing, China. Tel.: +86 67392010; Fax: +86 67392010; E-mail: yanglin@ bjut.edu.cn.
} 
Table 1

The screening results of the influence factors of GDM

\begin{tabular}{llcrcc}
\hline & Factors & Mean \pm SD & \multicolumn{1}{c}{$\chi 2$} & OR & P \\
\hline Epidemiology factors & AGE $(\geqslant 30)$ & $33.440 \pm 2.860$ & 46.003 & 5.091 & 0.000 \\
& BMI $(\geqslant 24)$ & $27.058 \pm 2.848$ & 74.436 & 5.055 & 0.000 \\
& DFH & - & 45.509 & 6.652 & 0.000 \\
& HFH & - & 22.245 & 2.295 & 0.000 \\
& AMH & - & 22.092 & 3.600 & 0.000 \\
& UOF & - & 5.184 & 4.412 & 0.000 \\
& MP & - & 7.627 & 4.801 & 0.006 \\
& PBH & - & 13.668 & 3.818 & 0.000 \\
Physical factors $(\Delta \mathrm{BMI})$ & BPI & - & 6.275 & 4.277 & 0.000 \\
& 16 20 week $(\geqslant 12)$ & - & 8.530 & 1.954 & 0.003 \\
Biochemical factors & $21 \sim 24$ week $(\geqslant 15)$ & - & 14.345 & 2.037 & 0.000 \\
& $25 \sim 28$ week $(\geqslant 18)$ & - & 6.191 & 1.537 & 0.013 \\
& WBC $\geqslant 10$ & - & 11.150 & 1.702 & 0.001 \\
& HBG $\geqslant 125$ & - & 23.431 & 2.179 & 0.000 \\
\hline
\end{tabular}

DFH: Diabetes Family History; HFH: Hypertension Family History; AMH: Adverse Maternal History; UOF: Uterine Ovary Fibroids; MP: Multiple Pregnancy; PBH: Pregnancy Bleeding History; BPI: Before Pregnancy Infection; WBC: White Blood Cells Count; HBG: Hemoglobin.

423 pregnant women were in the normal group, and 413 pregnant women were in the GDM group. The average age was $30.1 \pm 4.1$.

The standard of division was based on the new diagnostic criteria for GDM, which was proposed by the American Diabetes Association (ADA) in 2011 [12].

This study was based on the epidemiology factors, physical examination factors, and biochemical factors. Each factor was quantified with either " 0 " or " 1 ". Then, one-factor analysis of variance, chisquare test and U-test were used to sieve out the influence factors of GDM $(P<0.05)$. Next, a logistic regression model of binary classification was used to analyze these statistically significant risk factors that influence the occurrence of GDM. Finally, the OR value was obtained and used to determine the degree of contribution of the various factors to the occurrence of GDM. The logistic regression equation was as follows:

$$
\text { LogitP }=\text { Constant }+\mathrm{B} 1 \times \mathrm{X} 1+\mathrm{B} 2 \times \mathrm{X} 2+\mathrm{B} 3 \times \mathrm{X} 3+\mathrm{B} 4 \times \mathrm{X} 4 \ldots \mathrm{Bm} \times \mathrm{Xm}
$$

Through this equation, the $\mathrm{P}$ value to predict the morbidity was obtained:

$$
P_{\mathrm{GDM}}=\frac{e^{\text {LogitP }}}{1+e^{\text {LogitP }}}
$$

All statistical works were completed with SPSS18.0.

In this study, 23 epidemiology factors were chosen to analyze and screened using the method of chisquare. During the pregnancy, the weight growth rate of pregnant women was different, so according to the growth of the different periods of body mass index, the integral duration of pregnancy was divided into six gestational intervals: the $0 \sim 16$ week, $16 \sim 20$ week, $21 \sim 24$ week, $25 \sim 28$ week, $28 \sim$ 32 week, and week $33 \sim$ birth. The biochemical data from routine blood examinations of the pregnant women. One-factor analysis of variance and chi-square test were applied to screen $\Delta \mathrm{BMI}$ of gestational intervals and biochemical factors which were significantly influence GDM. All the screening results are showed in Table 1.

The comprehensive analysis was conducted in three phases. Each significant factor was quantified with either "0" or " 1 ". Then, the risk factors were input into the logistic regression model to proceed 
Table 2

Risk factors of gestational diabetes multivariate logistic regression analysis

\begin{tabular}{|c|c|c|c|c|c|c|c|c|c|}
\hline \multirow[t]{2}{*}{ Variable } & \multicolumn{3}{|c|}{$\frac{16 \sim 20 \text { week }}{\text { Constant: }-2.437}$} & \multicolumn{3}{|c|}{$\begin{array}{c}21 \sim 24 \text { week } \\
\text { Constant: }-1.682\end{array}$} & \multicolumn{3}{|c|}{$\begin{array}{c}25 \sim 28 \text { week } \\
\text { Constant: }-1.672\end{array}$} \\
\hline & B & $P$ & OR & $B$ & $\mathrm{P}$ & OR & B & $P$ & OR \\
\hline$X_{\text {DFH }}$ & 1.821 & 0.000 & 6.175 & 1.771 & 0.000 & 5.879 & 2.304 & 0.000 & 10.018 \\
\hline $\mathrm{X}_{\mathrm{AMH}}$ & 1.163 & 0.013 & 3.200 & 1.144 & 0.005 & 3.140 & 1.142 & 0.005 & 3.134 \\
\hline $\mathrm{X}_{\mathrm{UOF}}$ & 1.349 & 0.042 & 3.855 & 1.041 & 0.018 & 2.833 & 1.170 & 0.011 & 3.223 \\
\hline $\mathrm{X}_{\mathrm{MP}}$ & 2.003 & 0.025 & 7.411 & 1.742 & 0.039 & 5.711 & 1.899 & 0.022 & 6.676 \\
\hline $\mathrm{X}_{\mathrm{HFH}}$ & 1.149 & 0.002 & 3.156 & 0.732 & 0.022 & 2.080 & 0.887 & 0.009 & 2.427 \\
\hline $\mathrm{X}_{\mathrm{BMI}}$ & 1.317 & 0.000 & 3.733 & 1.343 & 0.000 & 3.829 & 1.576 & 0.000 & 4.835 \\
\hline $\mathrm{X}_{\mathrm{AGE}}$ & 2.088 & 0.000 & 8.067 & 1.838 & 0.000 & 6.286 & 1.756 & 0.000 & 5.791 \\
\hline $\mathrm{X}_{\mathrm{WBC}}$ & 0.680 & 0.016 & 1.975 & 0.000 & 0.000 & 0.000 & 0.000 & 0.000 & 0.000 \\
\hline $\mathrm{X}_{\mathrm{HBG}}$ & 0.571 & 0.045 & 1.770 & 0.511 & 0.021 & 1.668 & 0.501 & 0.029 & 1.650 \\
\hline $\mathrm{X}_{\triangle \mathrm{BMI}}$ & 1.100 & 0.000 & 3.003 & 0.651 & 0.004 & 1.917 & 0.772 & 0.002 & 2.165 \\
\hline $\mathrm{X}_{\mathrm{BPI}}$ & 0.000 & 0.000 & 0.000 & 1.757 & 0.044 & 5.795 & 1.640 & 0.060 & 5.157 \\
\hline $\mathrm{X}_{\mathrm{PBH}}$ & 0.000 & 0.000 & 0.000 & 0.587 & 0.054 & 1.799 & 0.000 & 0.000 & 0.000 \\
\hline
\end{tabular}

DFH: Diabetes Family History; AMH: Adverse Maternal History; UOF: Uterine Ovary Fibroids; MP: Multiple Pregnancy; HFH: Hypertension Family History: BMI: Body Mass Index; WBC: White Blood Cells Count; HBG: Hemoglobin; BPI: Before Pregnancy Infection; PBH: Pregnancy Bleeding History.

Table 3

The information of each ROC curve and the corresponding screening efficiency

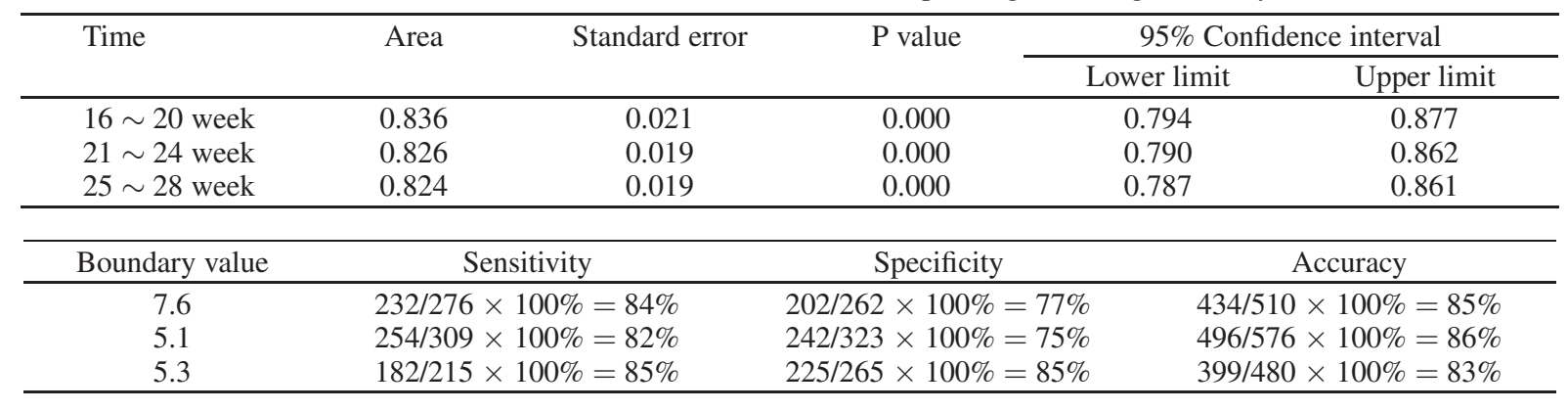

with the multi-factor logistic regression analysis. The OR values and $\mathrm{P}$ values of different gestational intervals are listed in Table 2. Finally, according to Eqs (1) and (2), the different gestational periods' corresponding logistic regression equation and $\mathrm{P}_{\mathrm{GDM}}$ were calculated out.

Certain scores based on the OR value of each factor were given to objectively evaluate the contribution of the risk factors to the different gestational periods. According to the score of each factor, pregnant women' risk score was calculated out. Then, the ROC curves of the scores in the three gestational periods were drawn. The ROC curves for each of the three intervals are shown in Fig. 1. In the figure, (1) is the ROC curve for the $16 \sim 20$ week, (1) for the $21 \sim 24$ week, and (3) for the $25 \sim 28$ week. The maximum value of the cut-off point of the sum of the sensitivity and specificity was selected as the boundary value to obtain the screening efficiency. The information of ROC curves and the screening efficiency of the score of each gestational period are shown in Table 3.

\section{Discussion}

The morbidity of GDM is currently on the rise. The increased number of cases is related to a convenient lifestyle. In large-scale surveys of pregnant women in China that were over the age of 30, pro- 


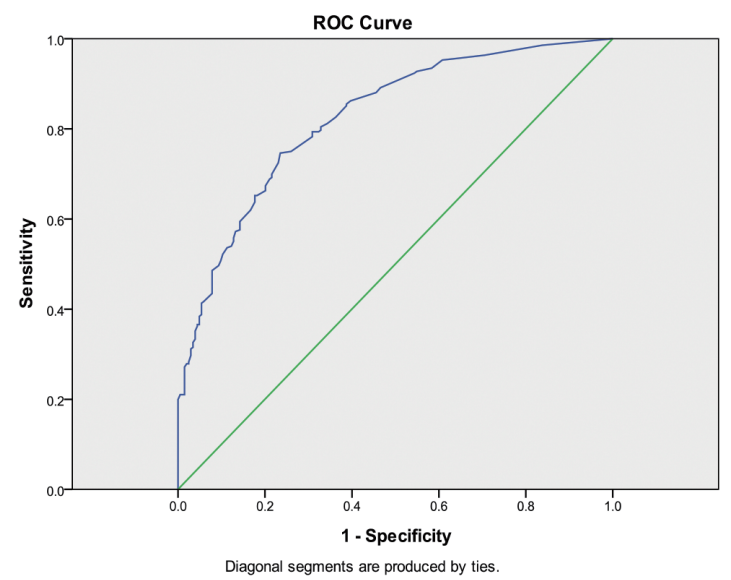

(1)

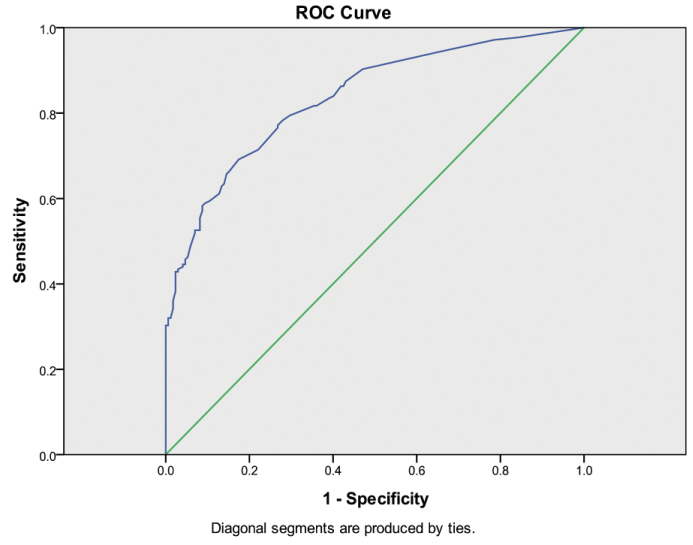

(2)

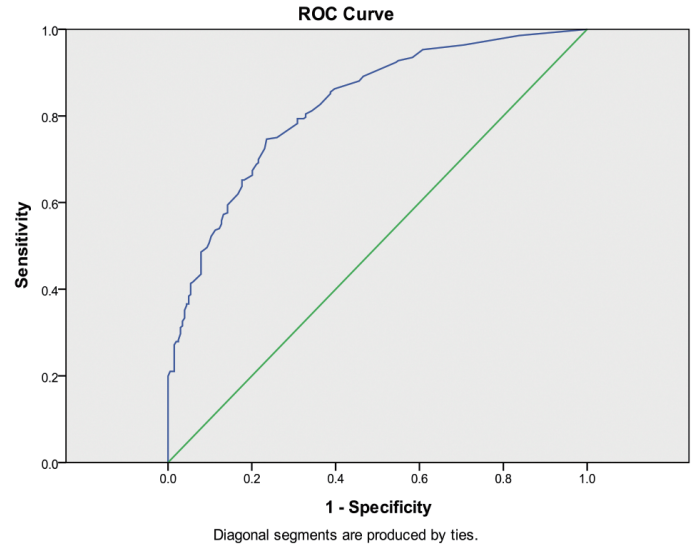

(3)

Fig. 1. The ROC curves of the comprehensive scores.

gestation obesity and a family history of diabetes are thought to be high risk factors of GDM [13-15]. Because of the gestation reaction, the weight gain of a pregnant woman is not obvious before the sixteenth week. The pregnant women whose OGT results are normal, in contrast, likely to continue their current lifestyle and usual diet, and consequently, their weight gain proceeds normally and does not slow down. This change of weight may have a little influence on the gravidas in the $29 \sim 32$ week interval, but after the thirty-second week, the normal group's BMI can overtake the GDM group's. Inflammatory reaction factors, such as the WBC and the C-reaction protein (CRP), are significantly related with pregnancy insulin resistance and are causally related to one another [16,17]. Several studies have found that when the prenatal examination is conducted, the higher the HBG concentration, the more likely GDM is to develop [18-20].

\section{Conclusion}

In this research, by combining the $\mathrm{P}_{\mathrm{GDM}}$ value with the comprehensive score to evaluate whether pregnant women will get GDM or not can be more reasonably and accurately and this study can greatly 
improve the prediction, detection, and intervention of GDM. Furthermore, this research has the potential to improve the quality of perinatal health care.

\section{Acknowledgements}

I would like to declare on behalf of my co-authors that the work described was not under consideration for publication elsewhere in whole or in part. All the authors listed have approved the manuscript that is enclosed.

\section{References}

[1] Z. Kerenyi, J. Toth, P. Stella. Follow-Up of Women with Previous Gestational Diabetes Mellitus: Reclassification and Frequency of Late Complications. Diabetologia. 1996.

[2] M.M. Hedderson, A. Ferrara. Rate of Gestational Weight Gain Before the Diagnosis of Gestational Diabetes Mellitus (GDM) and Subsequent Risk of GDM. Diabetes. 2009.

[3] W.T. Teh, H.J. Teede, E. Paul, C. Allan. Risk Factors for Gestational Diabetes Mellitus: Implications for the Application of Screening Guidelines. Australian \& New Zealand Journal of Obstetrics \& Gyanecology. 2011.

[4] M. Hod, D. Rabinerson, B. Kaplan, Y. Perinatal. Complications Following Gestational Diabetes Mellitus How 'Sweet' is Ill? Acta Obstetricia Et Gynecologica Scandinavica. 1996.

[5] Bellamy Leanne, Casas Juan-Pablo, Hingorani Aroon D, Williams David. Type2 diabetes mellitus after gestational diabetes: a systematic review and meta-analysis. The Lancet. 2009.

[6] Vambergue Anne. Gestational diabetes: Diagnosis, short and long term management. Presse Medicale. 2013.

[7] Reece E. Albert, Leguizamon Gustavo, Wiznitzer Arnon. Gestational diabetes: the need for a common ground. The Lancet. 2009.

[8] W.L. Bennett, K.A. Robinson, I.J. Saldanha, et al. High Priority Research Needs for Gestational Diabetes Mellitus. JOURNAL OF WOMENS HEALTH. 2012

[9] C.G. Solomon, W.C. Willett, V.J. Carey, et al. A Prospective Study of Pregravid Determinants of Gestational Diabetes Mellitus. JAMA-JOURNAL OF THE AMERICAN MEDICAL ASSOCIATION. 1997.

[10] C.G. Solomon, W.C. Willett, J. Richedwards, et al. Variability in Diagnostic Evaluation and Criteria for Gestational Diabetes. DIABETES CARE. 1996.

[11] S. Colagiuri, C. Lee, T.Y. Wong. Glycemic Thresholds for Diabetes-Specific Retinopathy Implications for Diagnostic Criteria for Diabetes. Diabetes Care. 2011.

[12] K. Rc. Gestational Diabetes Diagnostic Criteria: Long-Term Maternal Follow-Up. American Journal of Obstetrics And Gynetetrics. 1995

[13] B.E. Metzger, T.A. Buchanan, et al. Summary and Recommendations of the Fifth International Workshop-Conference On Gestational Diabetes Mellitus. DIABETES CARE. 2007.

[14] C.F. Qiu, I.O. Frederick, et al. Risk of Gestational Diabetes Mellitus in Relation to Maternal Egg and Cholesterol Intake. American Journal Of Epidemiology. 2011.

[15] Inegöl Gümüs Ilknur, et al. Association between prepregnancy body mass index, gestational weight gain, and perinatal outcomes. Turkish Journal of Medical Sciences. 2010.

[16] C.F. Qiu, M.A. Williams, S. Vadachkoria. Increased Maternal Plasma Leptin in Early Pregnancy and Risk of Gestational Diabetes Mellitus. American Journal of Obstetrics And Gynetetrics. 2004.

[17] E. Tarim, E. Kilicdag, T. Bagis, T. Ergin. High Maternal Hemoglobin and Ferritin Values as Risk Factors for Gestational Diabetes. International Journal of Obstetrics And Gynetetrics. 2004.

[18] C. Phaloprakarn, S. Tangjitgamol, S. Manusirivithaya. A Risk Score for Selective Screening for Gestational Diabetes Mellitus. European Journal of Obstetrics And Gynetetrics And reproducitive Biology. 2009.

[19] M.M. Hedderson, A. Ferrara. High Blood Pressure Before and During Early Pregnancy is Associated with an Increased Risk of Gestational Diabetes Mellitus. Diabetes Care. 2008.

[20] E. Tarim, E. Kilicdag, T. Bagis, T. Ergin. High Maternal Hemoglobin and Ferritin Values as Risk Factors for Gestational Diabetes. International Journal of Obstetrics And Gynetetrics. 2004. 